\title{
Primary Paediatric Renal Primitive Neuroectodermal Tumor: A Case Report and Review of the Literature
}

\author{
Binny KHANDAKAR, Moumita MAiti, Soumit DEY, Prasenjit Sen RAY, Palas BHATTACHARYYA, \\ Ranu SARKAR
}

Department of Pathology, Nil Ratan Sircar Medical College \& Hospital, KOLKATA, INDIA

\begin{abstract}
Ewing sarcoma/primitive neuroectodermal tumor (PNET) is a high-grade malignant neoplasm commonly affecting bones of the thoracic region. Primary Ewing sarcoma/PNET of the kidney is exceptional; it commonly affects young adults and is rarely reported in children.

Here we describe a case of renal PNET in a 10-year-old girl who presented at our institute with haematuria and abdominal lump. Computer tomography scan revealed a huge mass in the right kidney. Computer tomography-guided aspiration from the mass showed cytomorphological features of a small blue round cell tumor. The patient underwent right radical nephrectomy. Histopathology with supplementary immunohistochemistry confirmed the diagnosis of PNET. She has been receiving treatment with adjuvant chemotherapy post-surgery and is currently disease free.

Primary renal PNET is a distinct and rare entity. This tumor is very aggressive with low survival rate, even with a multi-disciplinary approach. We reported the case because it is rare in children, poses diagnostic challenges, is aggressive in behaviour and responds poorly to treatment.
\end{abstract}

Key Words: Ewing sarcoma, Kidney, Primitive neuroectodermal tumor

\section{INTRODUCTION}

Primitive neuroectodermal tumor (PNET) is a malignant neoplasm that originates from neural crest cells and the neuroectoderm that are found during normal human development. These tumors are broadly classified as central (CNS PNET) and peripheral, which is also known as Ewing sarcoma/primitive neuro-ectodermal tumor (ES/ PNET) due to its signature genetic alteration. ES/PNET is primarily a bone-soft tissue neoplasm. Primary ES/PNET of the kidney is an exceptionally rare malignant lesion (1). Median age of presentation is 27 years; it is extremely uncommon in $<15$ years and only a few sporadic cases have been reported so far (2). Conclusive diagnosis is done by morphology, immunohistochemistry (IHC) or molecular techniques. Patients are managed aggressively with surgery, chemotherapy with/without adjuvant radiotherapy. A commonly employed regimen includes combination chemotherapy with vincristine, adriamycin, cyclophosphamide (VAC) with alternating ifosfamide and etoposide (IE) (3). Response to treatment is often poor even with multimodality treatment. Overall prognosis and survival is mostly bleak though younger patients often have better survival than older ones (4). Here we report a case of primary ES/PNET of the kidney in a 10-year-old female.

(Turk Patoloji Derg 2018, 34:251-254)

Received : 26.08.2015 Accepted : 11.10.2015

\section{CASE REPORT}

A 10-year-old girl presented with intermittent haematuria for one week. Detailed clinical history revealed complaints of right-sided abdominal discomfort and generalised weakness for three months prior to the onset of haematuria. Examination revealed a palpable mass in the right lumbar region. Blood and urine examination showed mild anemia and presence of RBCs in the urine. Urine culture was negative. Serum creatinine was within normal limits. Contrast-enhanced Computer Tomography (CECT) Scan showed loss of normal right renal architecture and replacement with a huge mass $(10.44 \times 9.02 \mathrm{~cm})$, displaying hyper-dense enhancing and hypo-dense non-enhancing areas, without any excretion of contrast, suggesting a neoplastic lesion (Figure 1A). The left kidney was normal. A CT-scan guided aspiration from the mass showed cytomorphological features of a small blue round cell tumor. The patient subsequently underwent right radical nephrectomy.

Grossly, the specimen measured $14.5 \times 10 \times 8 \mathrm{~cm}$ and weighed $700 \mathrm{gm}$. The cut surface showed that almost the entire renal parenchyma was replaced by a solid, grey white tumor with areas of haemorrhage, necrosis, and cystic degenerations

Correspondence: Binny KHANDAKAR

Department of Pathology, Nil Ratan Sircar Medical College \& Hospital, KOLKATA, INDIA

E-mail: binikhandakar@gmail.com Phone: +91 3322653215 


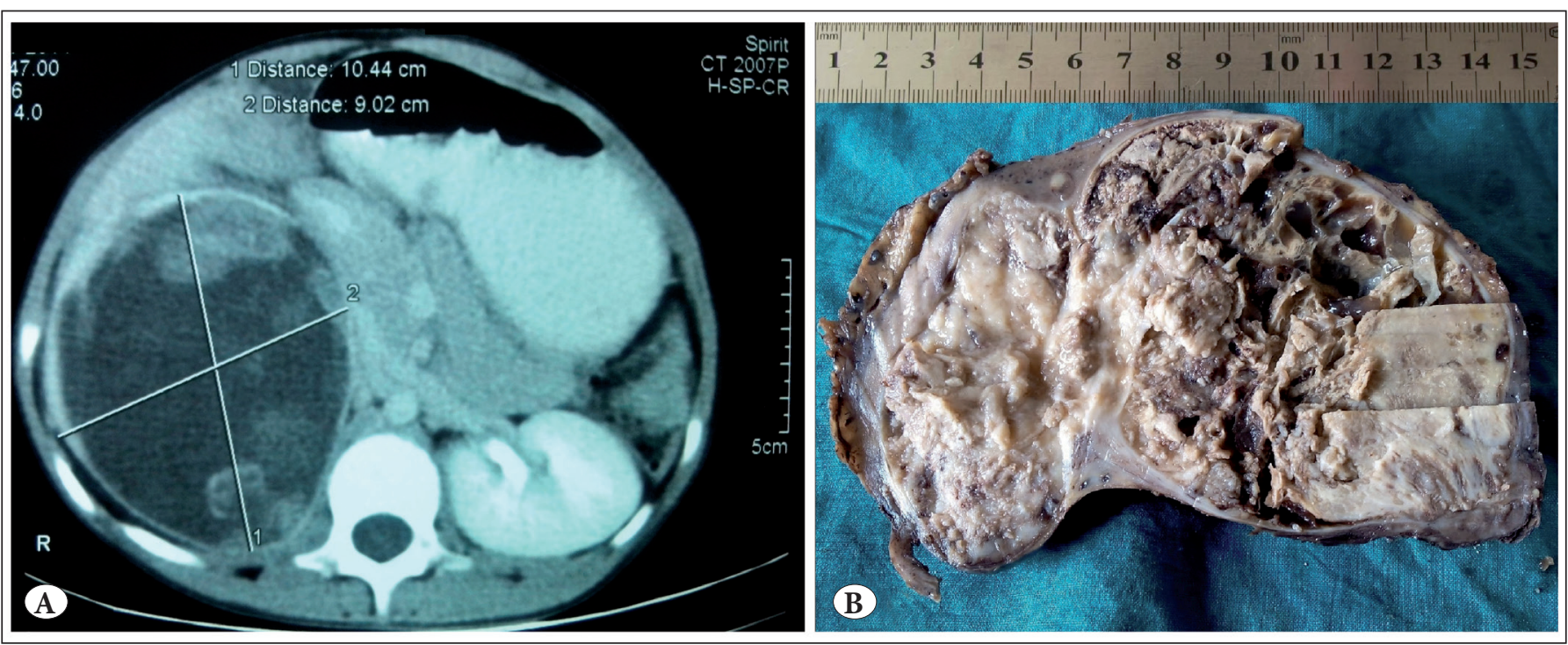

Figure 1: A) CECT image of right renal mass. B) Gross appearance of tumor with grey-white cut surface.

(Figure 1B). Multiple sections examined from the tumor showed tumor cells arranged in diffuse solid sheets, vaguely separated by broad fibrous bands, interspersed with perivascular pseudo-rosettes and haemorrhage at places (Figure 2A-C). The individual tumor cells were small and round with scanty vacuolated cytoplasm, and had round to oval vesicular to hyperchromatic nuclei, evenly dispersed granular chromatin, inconspicuous nucleoli and frequent mitosis. Large areas of necrosis and occasional lymphovascular emboli were also seen (Figure 2D). The renal sinus and perinephric fat showed involvement by the tumor, though the resected end of the ureter and hilar vessels and sampled lymph nodes were free of pathology. Periodic acid-Schiff (PAS) stain for intracytoplasmic glycogen revealed an occasional cell with minimal glycogen (Figure $2 \mathrm{E})$. A provisional diagnosis of small blue round cell tumor, possibly ES/PNET was made. A panel of IHC was ordered for chromogranin (CG), neuron-specific enolase (NSE), CD 99, Wilms' tumour (WT1), leukocyte common antigen (LCA), cytokeratin (CK), myogenin, desmin. The cells showed a strong and diffuse predominantly membranous expression of CD 99 (Figure 2F) with focal positivity for NSE; however the cells were immuno-negative for WT1, CG. Other makers which were negative included LCA (leukocyte common antigen), CK (cytokeratin), myogenin, desmin. Based on morphology and IHC results, a final diagnosis of renal PNET/ES was given. Molecular testing for the EWS-FLI-1 fusion gene or IHC for FLI1 expression could not be performed due to non-availability.

The patient had an uneventful post-operative period and was subsequently referred to the department of Oncology-
Radiotherapy for further management. She was started on chemotherapy four weeks post-surgery and has completed two cycles of chemotherapy without any obvious metastasis or recurrence.

\section{DISCUSSION}

PNET/ES covers one percent of all sarcomas (5). It is a malignant small round cell tumor of neural crest origin, first described in the ulnar nerve by Arthur Pourdy Stout in 1918 (6). Commonly, PNETs arise in the ribs and paraspinal areas; involvement of skin, soft tissue, lungs, kidney, and retroperitoneum is rare. Renal PNET was first described almost sixty years later than the first report of PNET, in 1975; few cases have been reported so far (7). Till now, around 100 cases have been reported worldwide, with very few from India (8).

Renal PNET commonly affects young adults, though the age range is $4-61$ years in reported cases. It more commonly affects males, with a male to female ratio of 3:1 (8). Most cases of renal PNET are diagnosed on resected specimens, based on histopathology and immunohistochemistry. The differential diagnosis includes other small round cell tumors, including Wilms' tumour, neuroblastoma, and lymphoma. These tumors are composed of primitiveappearing round cells with a high nucleo-cytoplasmic ratio. Perivascular pseudo-rosettes are usually identified; HomerWright rosettes are less frequently seen. A commonly appreciated feature on electron microscopy is aggregates of cytoplasmic glycogen granules though sometimes polar processes, microtubules or neurosecretory granules are seen, suggesting a neuronal differentiation (9). Tumor cells 


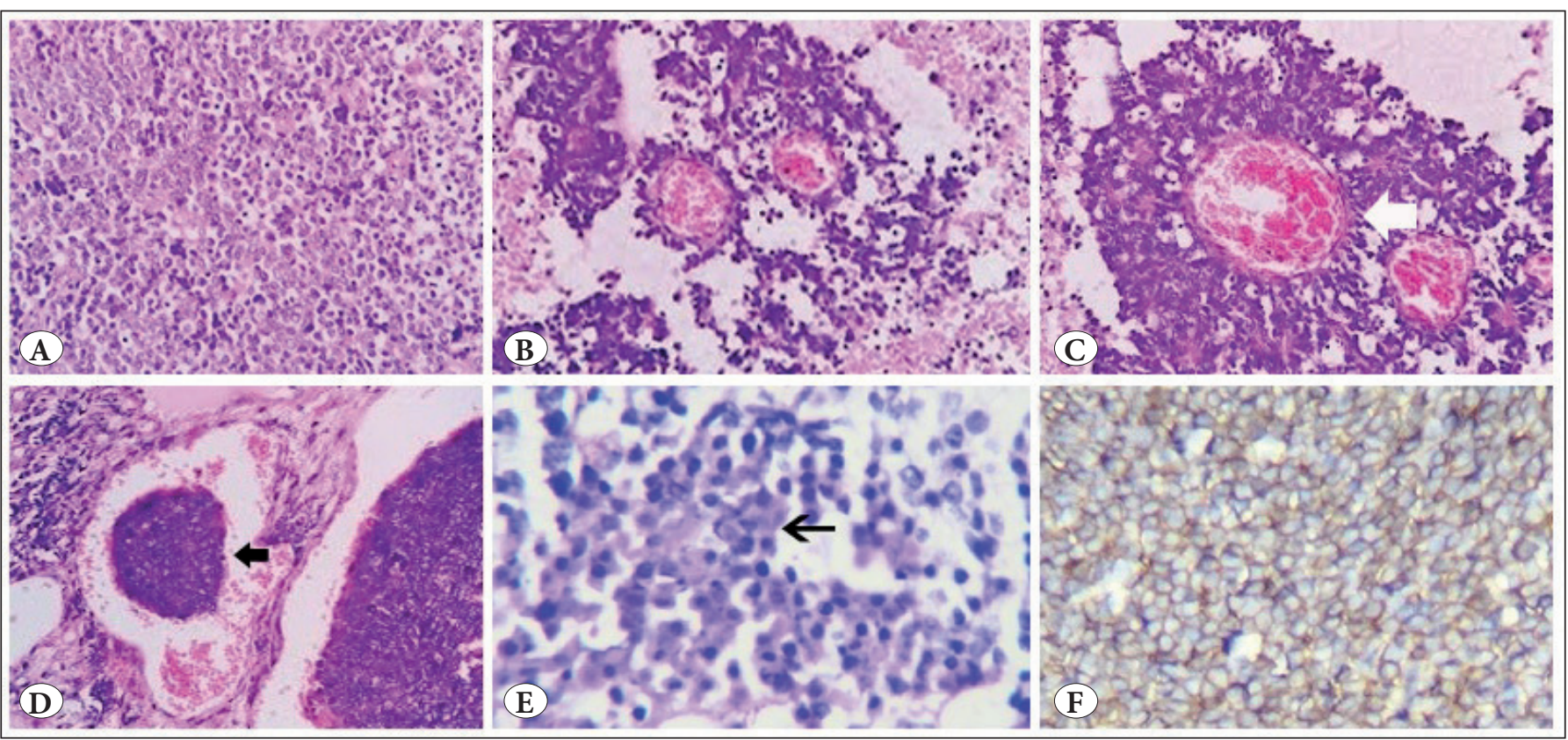

Figure 2: A) Tumor cells in diffuse sheets (H\&E; x40). B) Pseudo-rosette formation (H\&E; x40). C) High magnification of pseudo-rosette (white arrow) (H\&E; x100). D) Lymphovascular invasion (black arrow) (H\&E; x40). E) Minimal glycogen content in neoplastic cells (black arrow) (PAS; x100). F) Neoplastic cells showing CD99 positivity (CD99; x100).

express CD99 (MIC2) and FLI-1 with variable positivity for neuroendocrine markers including NSE, synaptophysin, and CG. WT1, a marker for Wilms' tumor, is not expressed in PNET. A combination of markers is generally helpful in arriving at the correct diagnosis. Eighty to ninety five percent of the cases show $t(11 ; 22)$ (q24;q12) while the remaining ones often display an EWS/Ets-related gene (ERG) mutation (10). Cytogenetic studies could not be performed in our case because of non-availability.

Radiological findings of renal PNET are nonspecific and commonly show a massive renal mass. Imaging finding of the present case included a large contrast-enhancing hyper- and hypo-dense mass without contrast excretion through the pelvi-calyceal system with very thin residual renal parenchyma.

As in other sites, renal PNET is very aggressive and about $50 \%$ patients come with distant metastasis at presentation. Common sites for metastasis are regional lymph nodes, liver, and lung. Overall survival is low and most patients do not live beyond one year following the diagnosis. Patients are generally treated with a multi-modality approach; radical nephrectomy with combination of chemotherapy with drugs including vincristine, doxorubicin, cyclophosphamide, etoposide and ifosfamide (9). Adjuvant radiation is given in patients with incomplete resection, positive resection margins, or recurrence.
In conclusion, primary renal PNET is a distinct and rare entity, typically affecting young adults. This tumor is very aggressive with a low survival rate, even with multimodality treatment. Although the incidence of renal PNET in children is low, oncologists and pathologists need to be aware of this tumor and every attempt should be made to differentiate it from other more common tumors as it carries very poor prognosis. Morphology alone can only suggest PNET as an important differential of small round cell tumors; ancillary techniques and immunohistochemistry for CD99 with or without a molecular test are vital to establish a correct diagnosis. We reported this case for its aggressive nature and rarity in children.

\section{REFERENCES}

1. Maeda M, Tsuda A, Yamanishi S, Uchikoba Y, Fukunaga Y, Okita $\mathrm{H}$, Hata J. Ewing sarcoma/primitive neuroectodermal tumor of the kidney in a child. Pediatr Blood Cancer. 2008;50:180-3.

2. Aghili M, Rafiei E, Mojahed M, Zare M. Renal primitive neuroectodermal tumor: Does age at diagnosis impact outcomes? Rare Tumors. 2012;4:e15.

3. Giridhar P, Mallick S, Kumaran D, George A, Kaushal S, Julka PK. Primitive neuro-ectodermal tumour of kidney in adult: Report of four consecutive cases and review of the literature. J Egypt Natl Canc Inst. 2015;27:235-8. 
4. Jimenez RE, Folpe AL, Lapham RL, Ro JY, O'Shea PA, Weiss SW, Amin MB. Primary Ewing's sarcoma/primitive neuroectodermal tumor of the kidney: A clinicopathologic and immunohistochemical analysis of 11 cases. Am J Surg Pathol. 2002;26:320-7.

5. Funahashi Y, Hattori R, Yamamoto T, Mizutani K, Yoshino Y, Matsukawa Y, Sassa N, Okumura K, Gotoh M. Ewing's sarcoma/ primitive neuroectodermal tumor of the kidney. Aktuelle Urol. 2009;40:247-9.

6. Sout AP. A tumour of the ulnar nerve. Proc N Y Pathol Sco. 1918;18:2-12.

7. Seemayer TA, Thelmo WL, Bolande RP, Wiglesworth FW. Peripheral neuroectodermal tumors. Perspect Pediatr Pathol. $1975 ; 2: 151-72$.
8. Mandal PK, Mukherjee S, Roy S, Bhattacharyya NK. PNET of kidney: Report of four cases. Indian J Med Paediatr Oncol. 2012;33:130-3.

9. Citak EC, Oguz A, Karadeniz C, Okur A, Akyurek N. Primitive neuroectodermal tumor of the kidney in a child. Pediatr Hematol Oncol. 2009;26:481-6.

10. Yang Y, Zhang L, Wei Y, Wang H, Xiong W, Chen Z, Hes O, Zheng J. Detection of EWSR1 translocation with nuclear extraction-based fluorescence in situ hybridization for diagnosis of Ewing's sarcoma/primitive neuroectodermal tumor. Anal Quant Cytol Histol. 2007;29:221-30. 